
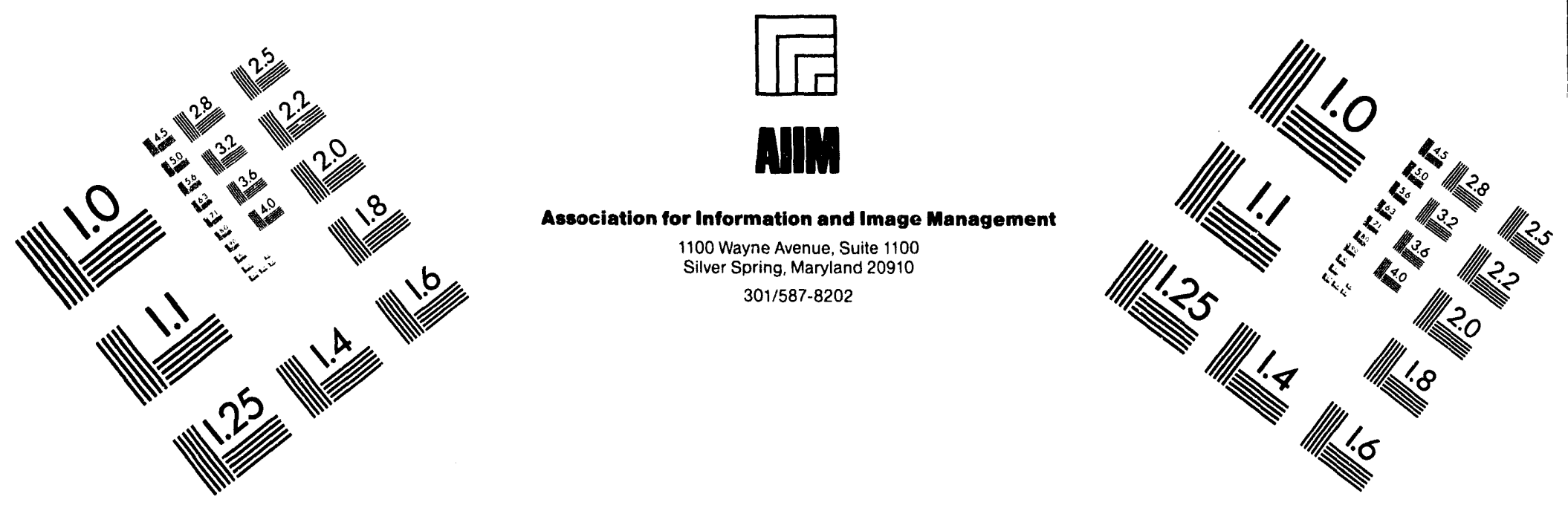

\title{
Centimeter
}

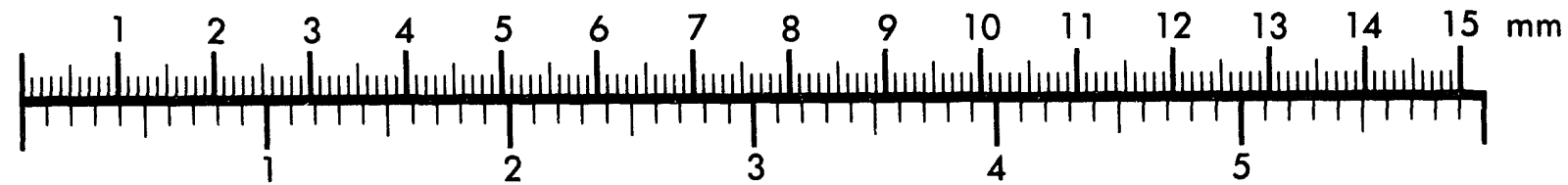

Inches
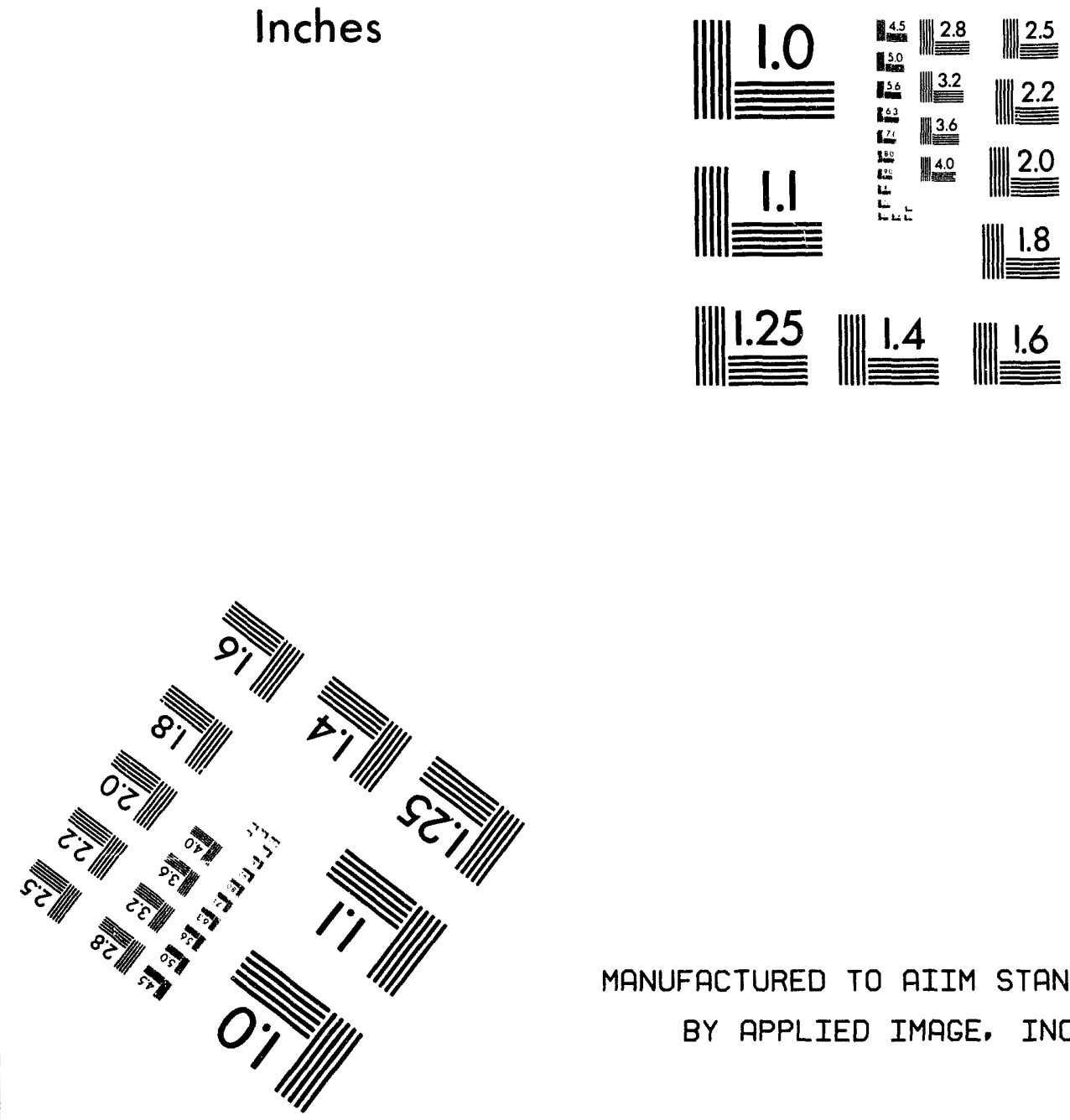

MANUFACTURED TO AIIM STANDARDS

BY APPLIED IMAGE, INC.

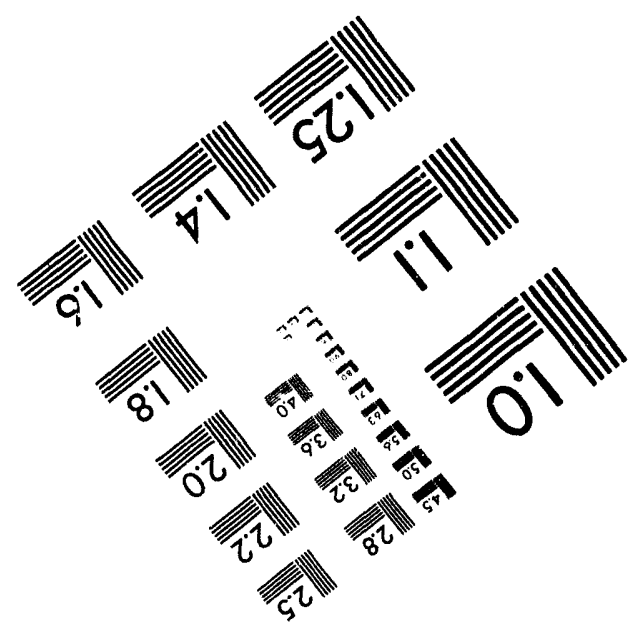



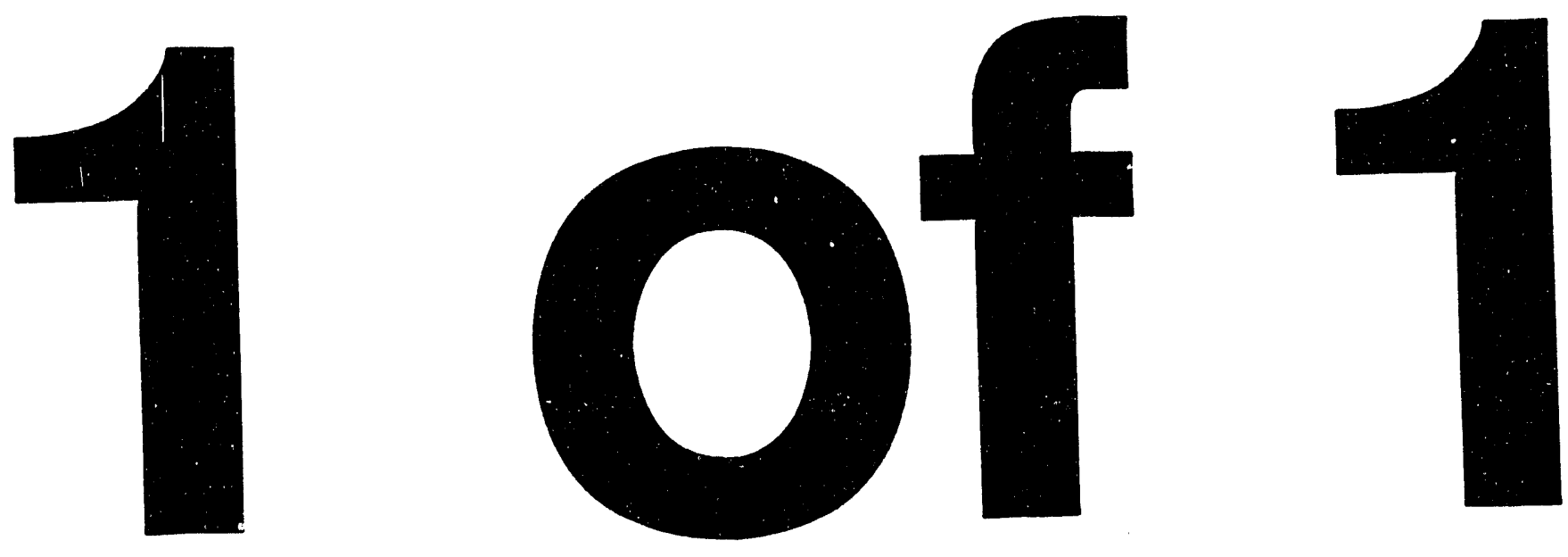
TECHNICAL REPORT $(F I N A L)$

\title{
MONOCHROMOSOMAL HYBRIDS FOR THE ANALYSIS OF THE HUMAN GENOME
}

\author{
RAGHBIR S. ATHWAL, PH.D. \\ Fels Institute for Cancer Research and Molecular Biology \\ Temple University School of Medicine
}

Grant Number: DE-FG02-89ER60866

\section{PROIECT SUMMARY}

The objective of this research project is to produce panels of mouse/human and/or Chinese hamster/human hybrid cell lines each harboring a single different human chromosome. The human chromosome present in rodent cell will be marked with a dominant selectable marker and maintained by selection.

In these experiments human chromosomes first "tagged" with a selectable marker in human cells are subsequetuly transferred to rodent cells by microcell fusion method. Several different experimental schemes have been developed to 'tag' human chromosomes with a selectable marker. Amphotropic retroviral vectors provide a highly efficient system to introduce selectable markers into normal diploid human cells. The integration of retroviral vector into the cell genome occurs at random by recombination at a defined nucleotide sequence in the LTRs and only a single copy of the vector integrates in a cell. This property of retroviral vectors allows to isolate a segment of the chromosomal DNA flanking the vector integration site by PCR amplification.

In these studies the amphotropic retroviral vector pZIPgpt that carries a dominant sulectable marker gpt, is used to tag the human chromosomes in normal diploid cells. Human DNA flanking the integrated vector is rescued by PCR amplification and cloned into a plasmid vector. Cloned human DNA is then used to probe Sc uthern blots of DNAs from a panel of hybrid cell lines to identify the chromosome of its origin. This allows us to identify clonal human cell lines, each carrying the marker integrated into a different chromosome. Marked chromosomes are then transierred to rodent cells by MMCT. Identification of the marked human chromosome in human cells prior to its transfer into rodert cells eliminates the redundancies in the recovery of the chromosomes in hybrid cells and thus will expedite the completion $c$. our hybrid cell panel. Once a chromosome is rescued in mouse cells, it can be transferred to Chinese hamster and/or other cell lines of interest.

Using the proposed experimental approach, we have already produced monochromosurial hybrids for 18 different human chromosomes which include $1,2,3,4,5,6,7,8,9,11,12,13$, $14,16,17,19,21$ and $X$. Each hybrid cell line is analyzed by cytogenetic and molecular methods to identify and determine the integrity of the human chromosome.

A panel of monuchromosomal hybrid cell lines will provide an invaluable biological resource for complementation analysis, cloning of chromosome specific DNA fragments, physical mapping of human chromosomes and fractionation of the human genome. The DNAs from hybrid cell lines will be made available freely for the analysis of human genome and gene mapping. 


\section{DISCLAIMER}

This report was prepared as an account of work sponsored by an agency of the United States Government. Neither the United States Government nor any agency thereof, nor any of their employees, makes any warranty, express or implied, or assumes any legal liability or responsibility for the accuracy, completeness, or usefulness of any information, apparatus, product, or process disclosed, or represents that its use would not infringe privately owned rights. Reference herein to any specific commercial product, process, or service by trade name, trademark, manufacturer, or otherwise does not necessarily constitute or imply its endorsement, recommendation, or favoring by the United States Government or any agency thereof. The views and opinions of authors expressed herein do not necessarily state or reflect those of the United States Government or any agency thereof. 


\section{A. OBJECTIVE}

The overall objective of our project is to transfer individual human chromosomes to rodent cells to generate a panel of hybrid cell lines each harboring a single different human chromosome (monochromosomal hybrid cell lines). The human chromosome present in rodent cells will carry a dominant selectable marker, Ecogpt, and will be retained by selection. We have already produced such hybrid cell lines for 18 different human chromosomes (Table 1). The future goals for the continuation of this program are:

1. Complete the panel of monochromosomal hybrid cell lines (i.e. mouse/human and for Chinese hamster/human) to represent all human chromosomes.

2. Analysis of all hybrid cell lines for chromosome identification and structural integrity. AluPCR - Southern blot method will be used to detect the presence of non-selectable chromosomes or chromosomal segments. In situ hybridization of Alu-PCR products from hybrid cell lines to metaphase spread of normal human cells will be used to determine the structural integrity of the transferred chromosome.

3. An extension of our program will be to generate a set of radiation hybrids for human chromoscme 2 each containing different segment of this chromosome. These hybrids will be used to construct a physical map of chromosome 2 .

Monochromosomal hybrids are used to map genes on human chromosomes and to construct physical maps. The materials produced in this project will serve as a national resource for various laboratories in the human genome project and are freely available to the research community. Hybrid cell lines for several chromosomes have already been made available to other laboratories for gene mapping studies.

\section{B. PROGRESS REPORT}

Since the inception of this project, we have produced monochromosomal hybrids for 18 different human chromosomes. We have also improved our methods and develop new techniques which will enhance our ability to fulfill our commitment to construct panels oi hybrid cell lines. During this budget period (1993-1994), we have produced 3 new hybrids which include chromosomes 3, 4 and 11. At the rate the project is now proceeding, we anticipate that the hybrid panels will be completed during this grant period. In addition, we have developed new techniques for the analysis of hybrid cell lines. Following is a summary of our progress to date:

\section{Monochromosomal Hybrids Produced to Date:}

The experimental approach used to produced monochromosomal hybrid cell lines is given in figure 1 and hybrids produced to date are listed in Table 1.

A retroviral vector carrying the selectable marker Ecogpt was introduced into the normal diploid human fibroblast cell line GM03468 and a total of 210 independent gene transfer clones were isolated by selection in MX medium. Normal human cells have a limited life span. In order to perpetuate the marked human chromosomes, gene transfer clones were fused with an immortal human tumor cell line HT1080TG (Fig. 1). In these studies, a total of 125 human $\mathrm{x}$ human hybrid cell lines have been produced.

As an intermediary step, clones of marked diploid human cells were also simultaneously hybridized with mouse A9 cells to generate mouse/human somatic cell hybrids (Fig. 1). Mouse/human hybrids are stored frozen while human/human hybrid clones are analyzed to identify the marked chromosomes. Once a marked chromosome is identified in human cells, the corresponding mouse/human hybrid cell line is used to transfer the marked chromosome to another recipient cell line by microcell fusion. 
Table 1: Monochromosomal hybrids containing marked human chromosomes.

Mouse/Human Hybrids

Human Chrom.

Independent Hybrid Cell Lines

RA-1

RA-2

RA-3

RA-4

RA-5

RA-6

RA-6q

RA-Iso 6q

RA-7

RA-8

RA-9

RA-11

RA-12

RA-12q

RA-13

RA-14

RA-15

RA-16

RA-17

RA-19

RA-21

RA-X

$\begin{array}{ll}1 & 2 \\ 2 & 5 \\ 3 & 3 \\ 4 & 2 \\ 5 & 3 \\ 6 & 3 \\ 6 \mathrm{q} & 3 \\ \text { Iso } 6 \mathrm{q} & 1 \\ 7 & 1 \\ 8 & 1 \\ 9 & 2 \\ 11 & 1 \\ 12 & 2 \\ 12 \mathrm{q} & 2 \\ 13 & 1 \\ 14 & 2 \\ 15 & 2 \\ 16 & 2 \\ 17 & 2 \\ 19 & 3 \\ 21 & 2 \\ \mathrm{X} & 1 \\ & 2\end{array}$

Hamster/Human Hybrids

Human Chrom.

Independent Hybrid Cell Lines

CHA-2

CHA-5

CHA-6q

CHA-6

CHA-13

CHA-15

$\begin{array}{ll}2 & 1 \\ 5 & 1 \\ 6 \mathrm{q} & 1 \\ 6 & 1 \\ 13 & 1 \\ 15 & 1 \\ \end{array}$

Mouse cell lines in general are easy to induce micronuclei formation. In addition, segregation of unmarked human chromosomes in whole cell hybrids facilitate the transfer of single chromosomes. Thus, mouse/human whole cell hybrid generation is an essential intermediary siep for the production of monochromosomal hybrids. Since our mission is to generate a panel of monochromosomal hybrid cell lines with marked chromosomes, hybrid cell lines containing unmarked single chromosomes and hybrids with two human chromosomes are not included in Table 1. For several chromosomes, more than one independent hybrid have been recovered.

Once a chromosome is rescued into mouse cells, it can be easily transferred to any other cell type of interest. We have already transferred several chromosomes to Chinese hamster cells to generate hamster/human monochromosomal hybrid cell lines (Table 1). 


\section{New Techniques Developed}

a. Identification of the Marked Chromosomes In Human Cells: (Fig. 2)

In the production of monochromosomal hybrid cell lines, we have been dependent upon fortuitous recovery of different chromosomes in microcell hybrids. Thus, hybrid cell lines for some chromosomes were obtained in duplicate or even triplicate while others were never recovered. The redundancy in the production of microcell hybrids can be reduced or even eliminated if the marked chromosomes can be identified in human cells prior to their transfer to mouse cells.

We have developed a new method using PCR technology to determine the identity of the marked chromosome in human cells. This will allow us to generate a bank of 24 human cell lines each carrying the selectable marker integrated into a different chromosome. The experimental strategy outlined in Fig. 2 involves PCR mediated amplification and cloning of the human DNA flanking the integrated vector. The cloned DNA is then mapped to a chromosome by Southern blot analysis of a panel of rodent/human hybrid cell lines.

The rationale for the cloning of the junction sequence is based upon the recombination dependent integration of the retroviral into cellular DNA involving a precise nucleotide sequence in LTRs (Fig. 2). Cellular DNA, isolated from the marked cells is digested with a frequent cutting restriction endonuclease which has unique sites in vector LTRs. As depicted in Fig. 2, SAU3A1 cuts each LTR into two segments resulting in four DNA fragments. In two of these fragments, part of the LTR is linked to the human cellular DNA flanking the integration site. Self ligation of the digested DNA, under conditions that promote circularization, yield unique DNA circles each containing a part of the LTR sequence. PCR amplification of ligated DNA with a set of oligonucleotide primers complementary to the end of 3' LTR yield DNA products comprised of $79 \mathrm{bp}$ vector DNA fragment and a variable length fragment of human DNA linked to a part of the LTR sequence. The human DNA product of PCR amplification is separated by gel electrophoresis and cloned into a plasmid. Cloned human DNA is used to probe the Southern blots of a panel of hybrid cell lines to determine the chromosome of its origin. Marked chromosomes identified in this manner are subsequently transferred to mouse cells by microcell fusion. The method will significantly expedite the production of monochromosomal hybrids. A manuscript describing the results of these experiments is currently being prepared for publication.

\section{b. Analysis of Hybrid Cell Lines}

The usefulness of monochromosomal hybrids depends upon the fact that only a single intact "tagged" chromosome is present in each cell line. Presence of chromoscmal fragments other than the marked chromosome or rearranged chromosomes would lead to erroneous interpretation of the data obtained with these hybrids. Thus, appropriate evaluation of each hybrid cell line is essential to ascertain the purity of the hybrid cell lines. Therefore, all hybrid cell lines are analyzed by several different methods.

We have developed a highly sensitive method to determine human chromosomal content of hybrid cells. In this method a 32P labeled probe, prepared for the Alu-PCR amplified DNA products of a new hybrid, is hybridized to the Southern blots of Alu-PCR amplified DNA from a hybrid cell panel (Fig. 3). Hybridization in this case occurs only with the DNA of the hybrid containing a corresponding human chromosome. As such, the method allows one to detect the presence of multiple chromosomes or chromosomal fragments in putative monochromosomal hybrid cell lines. All of the hybrids produced in our lab are subjected to this type of analysis. It helps to identify hybrids containing non-selectable chromosomal fragments or rearranged chromosomes. An Alu-PCR Southern blot that shows the presence of human chromosome 6 in a monochromosomal hybrid is shown in Fig. 3.

All of the hybrid cell lines are also analyzed by in situ hybridization using a total human DNA probe for chromosome painting to detect the presence of chromosomal fragments other than the marked chromosomes. Our results show that each of these hybrids contained only one human 
chromosome and no additional chromosomal fragments were observed.

\section{c. Fingerprinting Human Chromosomes}

Chromosome identification in hybrid cells is generally achieved by cytogenetic or molecular analysis for the presence of a limited number of markers. Both of these methods are inadequate when subchromosomal fragments or rearranged chromosomes are present in the hybrid cells. We have developed an approach for fingerprinting individual human chromosomes which allows the assignment of PCR amplified DNA fragments to the regions of a chromosome. A fingerprint map developed for a specific chromosome can also be used to identify chromosomal segments present in radiation-hybrids originating from a monochromosomal hybrid. A description of the method and its utility in gene mapping has been published in Genomics.

\section{d. Chromosome "Tagging" by Homologous Insertion}

We have developed a plasmid vector that carries human repeat sequences and the selectable markers NEO and HSV-TK. The vector when introduced into mouse/human hybrid cell lines, preferentially integrates into the human chromosome by homologous recombination. However, in addition to homologous insertion, random integration of the vector into mouse chromosomes also occurs. The vector is designed so that homologous insertion leads to the exclusion of HSV-TK gene while retaining the neo marker. On the other hand non-homologous insertion of the vector retains both NEO and TK genes. The cells expressing the neo marker can be selected by growth in medium containing G418. Addition of Gancyclovir (GANC) into the medium selects against HSVTK. Thus, hybrid cells in which the neo marker is integrated into a human chromosome by homologous insertion can be selectively separated by growth in medium containing G418 and GANC. We have used this method to insert neo marker into human chromosome 9 in hybrid RA9. Homologous insertion into human chromosome 9 occurred in $5 \%$ of the clones. We are using this method to insert a second marker into the human chromosomes already marked with gpt or to 'tag' unmarked human chromosomes present individually in hybrid cells. The presence of two or multiple markers in a human chromosome will provide a method to generate subchromosomal hybrids by double selection (i.e. for the retention of a marker and loss of the second marker) following X-irradiation. This approach will also expedite completion of hybrid cell panel.

\section{Publications}

The results of our research towards the production and use of the monochromosomal hybrids were presented in several conferences and published in a number of papers. A list of publications and meeting abstracts during this budget period is given here and a copy of each is enclosed with this report.

\section{a. Papers Published or Accepted (1992-1994)}

1. Sandhu, A.K., K. Hubbard, G.P. Kaur, K. Jha, H.L. Ozer and R.S. Athwal (1993). Senescence of immortal SV40-transformed human fibroblasts by the introduction of normal human chromosome 6. Proc. Natl. Acad. Sci. U.S.A. (In press).

2. Kansara, M.S. and R.S. Athwal (1993) Dinucleotide Repeat Polymorphism at D6S348 locus. Human Mol. Genetics 2: 7: 1085.

3. Jeggo, P., M. Hafezparast, A.F. Thompson, G.P. Kaur, A.K. Sandhu and R.S. Athwal (1993) Hamster-human hybrid cell lines bearing different fragments of human chromosome 2. Somatic Cell \& Mol. Genetics 19: 1: 39-49.

4. Kaur, G.P. and R.S. Athwal (1993) Complementation of DNA repair defect in XP-C by transfer of human chromosome 5. Somatic Cell and Molecular Genetics 19: 1: 83-93. 
5. Hafezparast, M., G.P. Kaur, M. Zdzienicka, R.S. Athwal, and P.A. Jeggo (1993) Subchromosomal localization of a gene (XRCC5) involved in double strand break repair to the region 2q 34-36. Somatic Cell and Mol. Genetics 19: 413-421.

6. Briggs, R.C., J. Briggs, J. Ozer, L. Sealy, L. Lane, S.F. Kingsmore, M.F. Seldin, G.P. Kaur, R.S. Athwal and E.N. Dessypsis (1993). The human myeloid cell nuclear differentiation antigen gene is one of at least two related interferon-inducible genes on chromosome $1 \mathrm{q}$ that are expressed specifically in hematopoietic cells. Blood (In press).

7. Jeggo, P.A., M. Hafezparast, A.F. Thompson, B.C. Broughton, G.P. Kaur, M. Zdzienicka, and R.S. Athwal (1992) Localization of a DNA repair gene (XRCC5) involved in double strand break rejoining to human chromosome 2. Proc. Natl. Acad. Sci. U.S.A. 89: 64236427.

8. Sidhu, M.S., B. Helen, and R.S. Athwal (1992) Fingerprinting human chromosomes by PCR mediated DNA amplification. Genomics 14: 728-732.

9. Kaur, G.P., A. Rinaldy, R.S. Lloyd, and R.S. Athwal (1992) A gene that partially complements defects in Xeroderma pigmentosum group A cells maps to human chromosome 8. Somatic Cell and Mol. Genet. 18: 4: 371-379.

10. Mori, T., T.L. Rinaldy, R.S. Athwal, G.P. Kaur, O. Nikaido, R.S. Lloyd and A. Rinaldy (1992) An XP complementation group A related gene: Confirmation using monoclonal antibodies against the Cyclobutante dimer and (6-4) photoproduct. Mutat. Res. 293: 2: 143150 .

\section{b. Manuscripts Submitted:}

1. Bagga, P.S. and R.S. Athwal (1993) Bacteriophage T4 gene denV complements DNA repair defect in a cockayne syndrome cell line. Mutation Res. (submitted).

2. Banga, S.S., K.T. Hall, A.K. Sandhu, D.T. Weaver and R.S. Athwal (1994) Complementation of V(D)J recombination defect and $x$-ray sensitivity of scid mouse cells by human chromosome 8 (submitted).

3. Conway, K., R.S. Athwal and M. Costa (1993) Induction of aneuploidy by nickel and chromium in a hybrid cell system. Mutation Res. (submitted).

\section{c. Manuscripts in Preparation:}

1. Athwal, R.S., A.K. Sandhu and G.P. Kaur. Monochromosomal hybrids for the genetic analysis of the human genome: Integration of a selectable marker and transfer of individual human chromosomes to rodent cells.

2. Kaur, G.P., A.K. Sandhu, K.D. Dobler, R. Day III and R.S. Athwal. Transfer of individual human chromosomes into tumor cells: Identification of a suppressor gene on chromosome 9 for malignant glioma.

3. Sidhu, M.S., A.K. Sandhu, and R.S. A.thwal. Mapping of Vector integration sites in human genome and transfer of the marked chromosomes to rodent cells by microcell fusion. 

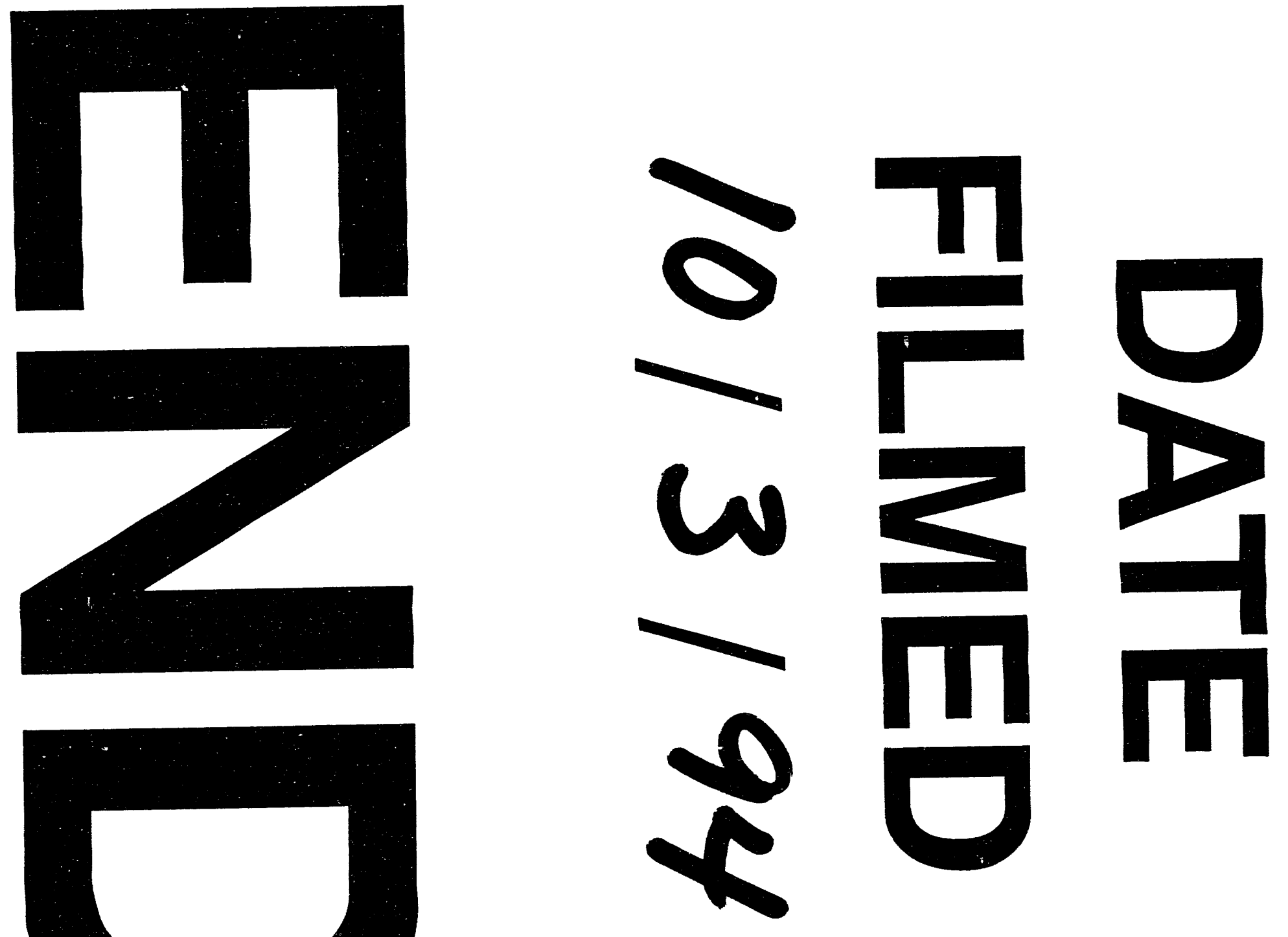
